\title{
Particle Sizing in the Process Industry using Hertz-Zener Impact Theory and Acoustic Emission Spectra
}

\author{
G. Carson*, A. J. Mulholland*, A. Nordon ${ }^{\dagger}$, M. Tramontana ${ }^{\ddagger}$, A. Gachagan ${ }^{\ddagger}$ and G. Hayward ${ }^{\ddagger}$ \\ * Department of Mathematics, University of Strathclyde \\ Livingstone Tower, 26 Richmond Street, Glasgow, UK, G1 1XH \\ ${ }^{\dagger}$ Department of Pure and Applied Chemistry \\ Centre for Process Analytics and Control Technology (CPACT) \\ University of Strathclyde, 295 Cathedral Street, Glasgow, UK, G1 1XL \\ $\ddagger$ Department of Electronic and Electrical Engineering \\ The Centre for Ultrasonic Engineering (CUE), University of Strathclyde \\ Royal College Building, 204 George Street, Glasgow, UK, G1 1XW
}

\begin{abstract}
The cost of implementing real-time monitoring and control of industrial processes is a significant barrier for many companies. Acoustic techniques provide complementary information to optical spectroscopic sensors and have a number of advantages: they are relatively inexpensive, can be applied non-invasively, are non-destructive, multi-point measurements are possible, opaque samples can be analysed in containers that are made from opaque materials (e.g. steel or concrete) and the analysis can be conducted in real-time.

In this paper a new theoretical model is proposed which describes the transport of particles in a stirred reactor, their collision with the reactor walls, the subsequent vibrations which are then transmitted through the vessel walls, and their detection by an ultrasonic transducer. The particle-wall impact is modelled using Hertz-Zener impact theory. Experimental data is then used in conjunction with this (forward) model to form an inverse problem for the particle size distribution using a least squares cost function. Application of an integral smoothing operator to the power spectra greatly enhances the accuracy and robustness of the approach. One advantage of this new approach is that since it operates in the frequency domain, it can cope with the industrially relevant case of many particle-wall collisions.
\end{abstract}

The technique will be illustrated using data from a set of controlled experiments. In the first instance a set of simplified experiments involving single particles being dropped in air onto a substrate are utilised. The second set of experiments involves particles in a carrier fluid being stirred in a reactor vessel. In each case the approach is able to successfully recover the associated particle size.

\section{INTRODUCTION}

The use of acoustic emissions as a process measurement technique has many advantages which are due in part to it being noninvasive and relatively inexpensive ([1], [2], [3], [4]). Several researchers have used time domain signals to determine particle sizes when powders are mixed, where the acoustic emissions have arisen from particle-particle interactions [5]. In this setting the sound is radiated into the surrounding air and recorded by a microphone [6]. Controlled experiments have also shown that time domain signals from particles colliding with a plate can be used to recover the size of a single impacting particle ([7], [8]). However, this approach depends on identifying the contact duration which is not practical in multi-particle, particle plate interactions such as in a stirred, particle laden fluid in a reactor vessel [9]. This problem can be circumvented by analysing the frequency domain acoustic emission spectrum. This has been conducted for the powder flow case (particle-particle interaction) and good agreement between the measured and theoretically derived spectra was achieved [10]. Both the single particle [11] and multi-particle [12] interaction with a plate have also been studied in this way. There have been some successful quantitative comparisons between the experimental and theoretical acoustic emission spectra generated by a single particle-particle interaction [13] and by multi-particle interactions ([14], [15]). However, a robust particle sizing methodology has yet to be devised which utilises this comparison for multi-particle interaction with a vessel wall.

Many of the studies to date have involved relatively simple experiments and do not address many of the practicalities of the industrial setting. This paper considers the case of a continuously stirred reactor vessel containing a particle laden fluid. To this end, a model of a particle being transported in a stirred vessel, its subsequent collision with the vessel wall and the detection of the sound emitted using an ultrasonic transducer attached to the exterior of the vessel is introduced. Analysis of the Hertz-Zener displacement response of the vessel wall leads to a new approximation for the form of the acoustic emission spectrum. Experimental data are shown which corroborates this analysis. A quantitative comparison is then conducted between the theory and experiment which leads to a least squares formulation for recovering the particle 


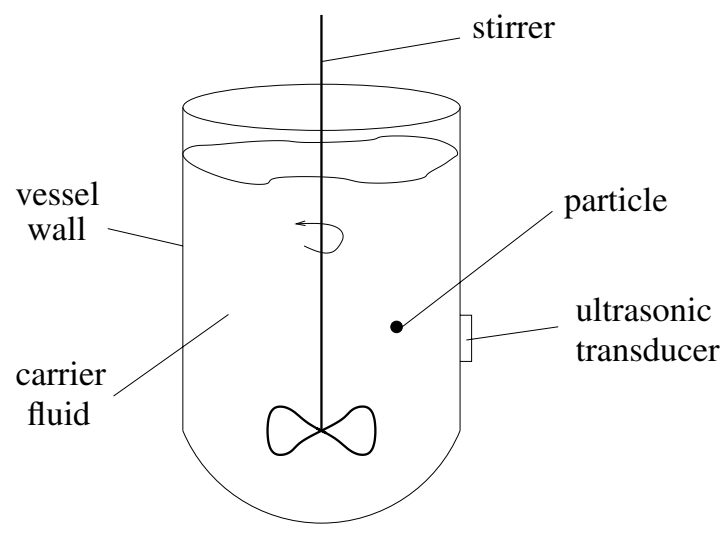

Fig. 1. Depiction of the model.

size. It transpires that smoothing of the respective spectra by integration leads to a successful particle size recovery methodology.

\section{PARTICLE MOVEMENT PRIOR TO IMPACT}

The first stage is the derivation of a simple model of the particle movement in the vessel. This restricts the particle motion to the radial direction only, with the centrifugal force being balanced by particle drag and a boundary layer effect near the wall surface. This component of the model provides the velocity of impact needed for the subsequent vibrational analysis. The approximate impact velocity $u_{0}[16]$ is given by

$$
u_{0}=\frac{\partial x}{\partial t}=\frac{2 b^{2} \omega^{2}(R-\epsilon)\left(\rho_{p}-\rho_{f}\right)}{9 \varphi(1+2 g b / \epsilon)},
$$

where $R$ is the plate radius, $b$ is the particle radius, $\omega$ is the stirrer rotation speed, $\varphi$ is the fluid viscosity, $g$ is a geometrical constant (2.104 for a sphere), $\rho_{p}$ and $\rho_{f}$ are the densitites of the particle and fluid respectively, and $x=R-\epsilon$ where $x$ is the displacment and $\epsilon \ll 1$.

\section{APPROXIMATE SOLUTION TO Plate RESPONSE}

A series expansion in the internal loss factor results in an approximation to the plate response function ([17], [16]). This then facilitates an investigation of the dependency of the acoustic emission spectrum on the particle size. A Fourier analysis of this approximation then produces an analytic expression for the frequency domain spectrum. Further algebraic manipulations demonstrate the explicit dependency of the first peak of the power spectrum, $\omega_{\max }$, on the properties of the particle, plate and carrier fluid [16]

$$
\omega_{\max }=\frac{9 \pi^{2}-104+\sqrt{81 \pi^{4}-144 \pi^{2}-4544}}{2\left(9 \pi^{2}-80\right)} \omega_{0},
$$

where

$$
\begin{gathered}
\omega_{0}=\frac{\pi}{2.9432}\left(\frac{4}{5 \pi}\right)^{2 / 5} \frac{u_{0}^{1 / 5}}{\left(\rho_{p} z\right)^{2 / 5}} \frac{1}{b}, \\
z=\frac{\left(1-\nu_{1}^{2}\right)}{E_{1}}+\frac{\left(1-\nu^{2}\right)}{E},
\end{gathered}
$$

TABLE I

EXPERIMENTAL SINGLE PARTICLE SIZES COMPARED WITH THEIR RECOVERED VALUES

\begin{tabular}{|c||c|}
\hline Actual size $(\mu \mathrm{m})$ & Approx. Recovered Size $(\mu \mathrm{m})$ \\
\hline $850-1000$ & 800 \\
\hline $500-600$ & 700 \\
\hline $400-500$ & 600 \\
\hline $200-300$ & 590 \\
\hline
\end{tabular}

$E, E_{1}, \nu$ and $\nu_{1}$ are the Young's Moduli and Poisson's Ratio of the particle and plate materials respectively, $u_{0}$ is the impact velocity, $b$ is the particle radius and $\rho_{p}$ is the particle density. Thus, as the particle size increases, the first peak in the acoustic spectrum $\omega_{\max }$ should shift to a lower frequency. In a similar way, as the particle density increases, the first peak should move to a lower frequency, and as the particle velocity is increased, $\omega_{\max }$ should translate to a higher frequency. This latter effect will be far less pronounced of course.

\section{EXPERIMENTAL RESUlts}

In order to test the model, a series of controlled experiments were performed by the Centre for Process Analysis and Control Technology (CPACT) [16].

\section{A. Single-particle experiments}

Experiments were performed using glass beads with size categories of $200-300 \mu \mathrm{m}, 400-500 \mu \mathrm{m}, 500-600 \mu \mathrm{m}$ and $850-1000 \mu \mathrm{m}$. The beads were repeatedly dropped from different measured heights onto the centre of a glass plate attached to a transducer, and the response recorded.

To quantitatively compare the model with the experiment and to facilitate the recovery of the particle size using a least squares method, it proved expedient to smooth the experimental data by integrating the spectrum over a frequency range. This process produces a clear delineation between the data assiciated with each particle size category and also ranks them in the correct order except for the two smaller sizes $400-500 \mu \mathrm{m}$ and $200-300 \mu \mathrm{m}$ [16]. A least squares minimisation method is then applied to automate the inverse problem of recovering the particle size. The least squares formula is explicitly

$$
p(b)=\sum_{i=1}^{N}\left\|E\left(\omega_{i}\right)-M\left(\omega_{i}, b\right)\right\|,
$$

where $E$ is the integrated experimental frequency response, $M$ is the integrated theoretical model frequency response and $b$ is the particle size. A plot of $p(b)$ shows clearly defined minima in all cases and the location of these minima is then used to estimate the particle size [16].

Table I shows the recovered particle sizes that are obtained. It can be seen that there is encouraging agreement with the actual 


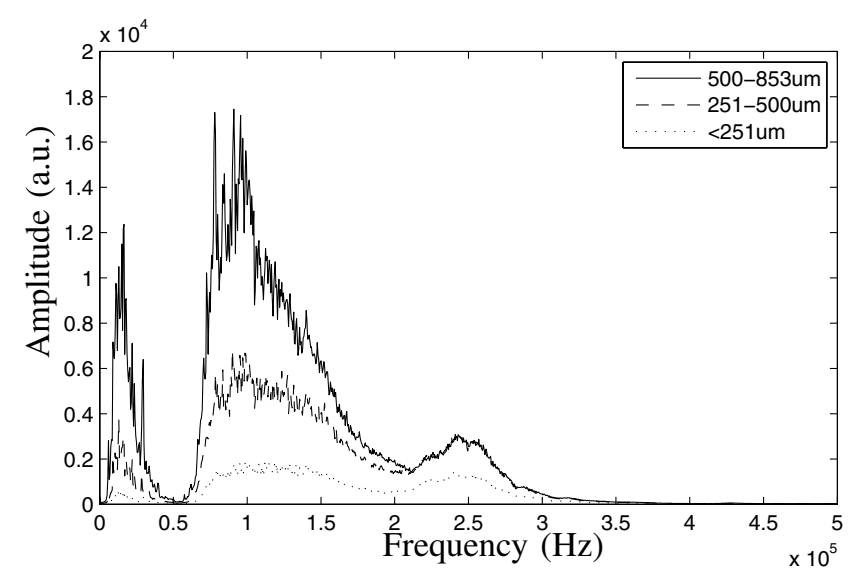

Fig. 2. Measured power spectrum arising from itaconic acid-toluene experiments.

sizes, particularly for the larger particles. The difficulty in estimating the smaller particle sizes stems from the similarities found in their experimentally measured power spectra. Despite this, the method still manages to rank the particles in the correct order. Considering the simplicity of this initial model, the results are extremely encouraging and compare well with the experiment.

\section{B. Multi-particle experiments}

A series of multi-particle experiments have also been performed and a similar methodology applied. Itaconic acid was stirred in toluene in a 1 litre glass bell-shaped vessel with a glass stirrer rod and paddle, attached to a motor. Three specific size categories were utilized: $<251 \mu \mathrm{m}, 251-500 \mu \mathrm{m}$ and $500-853 \mu \mathrm{m}$. The signals from the particle-wall collisions were then collected using an ultrasonic transducer directly adhered to the side of the vessel as shown in Figure 1. The particle impact velocity was calculated using (1). The experimental power spectrum shown in Figure 2 shows agreement with our theoretical prediction in that the main lobe shifts to a lower frequency as the particle size is increased. It transpires that the integrated multi-particle data for each of the three particle size categories are once again sufficiently segregated and correctly ranked [16]. Applying the least squares methodology shows that very clear minima appear in the cost function space. The estimated particle sizes are also correctly ranked according to their particle size categories and have reasonable agreement with the actual values (see Table II). To conclude, both the single-particle and the multi-particle experiments agree with the theoretical prediction that the main spectral lobe shifts to a lower frequency as the particle size increases. In addition, a methodology has been presented that can quickly and accurately recover the approximate size of the impacting particles.

\section{CONCLUSION}

A model has been constructed for particles being transported in a stirred vessel, the subsequent impact with the vessel
TABLE II

EXPERIMENTAL MULTI PARTICLE SIZES COMPARED WITH THEIR RECOVERED VALUES

\begin{tabular}{|c||c|}
\hline Actual size $(\mu \mathrm{m})$ & Approx. Recovered Size $(\mu \mathrm{m})$ \\
\hline $500-853$ & 400 \\
\hline $251-500$ & 290 \\
\hline$<251$ & 220 \\
\hline
\end{tabular}

wall and the resulting acoustic vibrations which are emitted. An analytic approximation for the vessel wall vibrations has been developed and implemented, and a series of experiments have been carried out which have then been compared with this model. A least squares method was then applied in the frequency domain with the goal of solving the inverse problem of recovering the particle size from the experimental data. Theoretical predictions were made by the model and were then compared with the data. Results show that the model and experiment compare very favourably considering the simplicity of the model. There are many improvements that could be made to the model in the future. For example, the inclusion of fluid loading effects, the incorporation of the vessel geometry, or consideration of a particle size distribution.

\section{ACKNOWLEDGMENTS}

The authors would like to thank the EPSRC for their funding support and the Royal Society for the award of a University Research Fellowship to AN.

\section{REFERENCES}

[1] M.J.W. Povey and T.J. Mason, Ultrasound in food processing, Blackie Academic and Professional, London, UK, 1998.

[2] J.R.W. Boyd and J. Varley, The uses of passive measurement of acoustic emissions from chemical engineering processes, Chemical Engineering Science, 56, pp1749-1767, 2001.

[3] G.P. Hancke and R. Malan, A modal analysis technique for the on-line particle size measurement of pneumatically conveyed pulverized coal, IEEE Transactions on Instrumentation and Measurement, 47(1), pp114122, February 1998.

[4] P.L. Lee, R.B. Newell and I.T. Cameron, Process control and management, Blackie Academic and Professional, London, UK, 1998.

[5] M.F. Leach, G.A. Rubin and J.C. Williams, Particle size determination from acoustic emissions, Powder Tech., 16, pp153-158, 1977.

[6] J. Hidaka, A. Shimosaka and S. Miwa, The effects of particle properties on the parameters of impact sound between two particles, KONA, 7, pp4-14, 1989.

[7] P. Troccaz, R. Woodcock and F. Laville, Acoustic radiation due to the inelastic impact of a sphere on a rectangular plate, J. Acoust. Soc. Am. 108(5) Pt.1, pp2197-2202, November 2000.

[8] D.J. Buttle and C.B. Scruby, Characterization of particle impact by quantitative acoustic emission, WEAR, 137, pp63-90, 1990.

[9] A. Nordon, Y. Carella, A. Gachagan, D. Littlejohn and G. Hayward, Factors affecting broadband acoustic emission measurements of a heterogeneous reaction, Analyst 131, pp323-330, February 2006.

[10] J. Hidaka and A. Shimosaka, Parameters of radiated sound and state variables in flowing particles, Intl. J. Mod. Phys. B, 7(9\&10), pp1965$1975,1993$.

[11] D. Takahashi, Frequency analysis of sound radiation from an impactexcited plate, J. Acoust. Soc. Am.19(5), pp 2708-2713, May 1992.

[12] J. Hidaka, A. Shimosaka, H. Ito and S. Miwa, Instantaneous measurement of particle size and flow rate by the parameters of impact sound between particles and a circular plate, KONA, No.10, pp175-183, 1992. 
[13] P.D. Thorne and D.J. Foden, Generation of underwater sound by colliding spheres, J. Acoust. Soc. Am 84(6), pp2144-2152, 1988.

[14] P.D. Thorne, The measurements of acoustic noise generated by moving artificial sediments, J. Acoust. Soc. Am. 78(3), pp1013-1023, September 1985.

[15] P.D. Thorne, Laboratory and marine measurements on the acoustic detection of sediment transport, J. Acoust. Soc. Am. 80(3), pp899-910, September 1986.
[16] G. Carson and A.J. Mulholland, Particle sizing using Hertz-Zener impact theory and acoustic emission spectra, Research Report No.15, Department of Mathematics, University of Strathclyde, Glasgow, UK, September 2005.

[17] A. Akay and M. Latcha, Sound radiation from an impact-excited clamped circular plate in an infinite baffle, J. Acoust. Soc. Am. 74(2), pp640-648, August 1983. 